\title{
Infectious Complications After Allogeneic Hematopoietic Stem Cell Transplantation in Children in a Bone Marrow Transplant Unit in Colombia
}

\author{
Ana Bravo ${ }^{1}$, Jailer Arango ${ }^{2}$, Oscar Ramirez ${ }^{3}$, Carlos Portilla $^{4}$, Pio López ${ }^{5}$, Juan \\ Calle-Giraldo ${ }^{6}$, and Eduardo Lopez-Medina ${ }^{5}$ \\ ${ }^{1}$ Universidad del Cauca \\ ${ }^{2}$ Universidad del Valle \\ ${ }^{3}$ Registro Poblacional de Cáncer de Cali \\ ${ }^{4}$ Escuela de Medicina, Universidad del Valle, Cali, Colombia \\ ${ }^{5}$ Centro de Estudios en Infectología Pediátrica \\ ${ }^{6}$ Universidad del Quindío
}

June 26, 2020

\begin{abstract}
To address the relative lack of information about infections occurring in children following allogeneic hematopoietic stem cell transplants (allo-HSCT) in developing countries, we performed a retrospective cohort study of 139 children who underwent 150 allo-HSCTs in a hospital in Cali, Colombia. Most allo-HSCT $(n=144,96 \%)$ were followed by infections over the following year, primarily due to bacteria (4.3 per 1000 patient-days) and CMV (3.3 per 1000 patient-days). Most infections occurred in the first 30 days post HSTC. High mortality $(\mathrm{n}=44,31.7 \%)$ was associated with infections, but has declined annually from $40 \%$ to $17 \%$ over the period covered (2012-2017).
\end{abstract}

\section{Introduction}

Children undergoing hematopoietic stem cell transplants (HSCT) suffer from profound immunosuppression that varies in intensity and duration according to the post-transplant period. ${ }^{1}$ Rates of HSCT-related bacterial, viral and fungal infections have been well described in industrialized countries but this epidemiology varies according to geographic, social and cultural determinants. ${ }^{2,3}$ There is a paucity of data in children who develop HSCT-related infections in developing countries.

A detailed description of overall rates of different infections during the HSCT periods according to the type of underlying disease, allows clinicians to narrow the differential diagnosis, apply targeted diagnostic strategies and institute prompt therapies while practicing judicial use of antibiotics.

Herein, we describe the epidemiology of infections in a cohort of children receiving an allogeneic HSCT (allo-HSCT) in a hospital in Cali, Colombia.

\section{Methods}

\section{Study design and participants}

This is a cohort study with retrospective collection of data of all pediatric patients who received at least one allo-HSCT at Centro Médico Imbanaco in Cali, Colombia from January 1, 2012, the beginning of the 
transplant program, until March 30, 2018. We reviewed medical records from cell infusion (day 0) until day +365 post-HSCT. The study was approved by the institutional review board.

\section{Diagnostic and therapeutic strategies}

Diagnoses and therapeutic strategies were performed according to physician's preference, adhering to international guidelines. ${ }^{4,5}$ At least one set of blood cultures was performed when a systemic infection was suspected. Laboratory equipment for blood cultures varied throughout the study period; most processing used a BD Bactec 9050? (identification by MALDITOF, bioMerieux since 2014) and susceptibility testing was done in a VITEK2? (bioMerieux).

For cytomegalovirus (CMV), preventative strategies with weekly serum polymerase chain reaction (PCR, Cobas? system, Roche) were implemented throughout the first 100 post-transplant days, or until required by clinical condition. Positive results triggered further treatment and evaluation. ${ }^{6}$ For adenovirus (AdV) infection, a preventive strategy was started in 2014 that was performed while the patients were hospitalized with weekly stool viral load assessment (Cobas? system, Roche). ${ }^{7}$ If more than 1,000,000 copies were detected in stool, the blood viral load was measured and cidofovir was considered. ${ }^{8}$ From February, 2015 we performed a preventive strategy for toxoplasma with bi-weekly blood PCR (Cobas? system, Roche) while the patients were hospitalized.

Evaluation of respiratory infections was done by immunofluorescence until June 2017, after which the FilmArray? respiratory panel was used.

From January 2017, all patients had rectal screening in the pre-HSCT period for enterobacteriaceae producing extended spectrum beta-lactamases or with reduced susceptibility to carbapenems with CHROMAgar? KPC with identification by VITEK? MS, bioMerieux. Results of rectal screening were taken into consideration when deciding empiric antibiotics.

\section{Prophylaxis}

Acyclovir, trimethoprim sulfamethoxazole and antifungal prophylaxis were given according to patient's age and risk factors. ${ }^{1,4,9}$

\section{Definitions}

Timing of infection after HSCT was divided into three periods, as described previously: 0-30, 31-100, and 101-365 days. ${ }^{1,4,10}$ We only include microbiologically-confirmed bacterial infections. Multidrug-resistant (MDR) or extensively drug resistant (XDR) bacterial infections, ${ }^{11}$ fungal infections, ${ }^{12} \mathrm{CMV}^{13}$ or $\mathrm{AdV}^{14}$ infections were defined according to internationally accepted criteria. Deaths were considered infectionrelated according to microbiologic reports and the working diagnosis in the transplant physician and infectious disease consultation notes.

\section{Results}

Of 139 patients who received 150 allo-HSCT, $64 \%$ were male, mean age was 9.6 years (SD 4.9), 87 (63\%) were of mixed race, $28(20 \%)$ were Colombian natives and $23(18 \%)$ were afro-descendants. Main reasons for the allo-HSCT were leukemia $(\mathrm{n}=83,59 \%)$, aplastic anemia, $(\mathrm{n}=32,21 \%)$ and hemoglobin disorders $(\mathrm{n}=21$, $14 \%)$. There were $76(49 \%)$ haploidentical transplants, $42(28 \%)$ matched sibling donors and $32(21 \%)$ transplants from umbilical cord blood. An infection developed after 144 (96\%) allo-HST cases during the first year.

Rectal screenings were performed in 97 allo-HSCT. The positive and negative predictive values for the bacteria that were screened were $10 \%(4-23 \%)$ and $91 \%$ (86-94\%), respectively.

During the study period, the total exposure time for the 150 allo-HSCT was 43,994 patient-days (Table 1). All infections, except respiratory infections, had higher incidence rates in the first period. Throughout the study period, bacterial and CMV infections were the most common, especially during the first period. 
Overall mortality was $32 \%$, with a higher frequency in the third period (Table 1). All deaths in the first period were associated with infectious diseases, although the proportion of infection-related deaths decreased in later periods $(100 \%, 67 \%$ and $45 \%$, respectively, $\mathrm{p}<0.001)$. Overall mortality decreased annually from transplants performed in 2012 until 2017 (40\%, 38\%, 32\%, 30\%, 23\%, 17\%).

Except for invasive fungal infections, the rates of different infections, overall mortality and infection-related mortality was similar in the different categories of underlying diseases (Table 2).

\section{Discussion}

In this series, $96 \%$ of children developed at least one infection during the first year of follow-up after HSCT. During the first period, as previously reported, ${ }^{15}$ bacterial infections represented the highest risk for patients, probably related to disruption of innate immune barriers and profound neutropenia. ${ }^{1}$ MDR and XDR bacterial infections were a significant burden for this cohort, especially during the first two periods, causing $71 \%$ and $77 \%$ of gram-negative infections, respectively. XDR bacteremia is a major threat during the first two periods, as $20 \%$ and $56 \%$ of these infections resulted in fatal outcomes, respectively.

Similarly, there was a high incidence of viral infections, especially due to CMV, during the first two periods although the rate of disease was stable throughout the study period. Adenovirus infections, although less common than CMV, were a frequent cause of mortality highlighting the need for better therapeutic strategies.

We report a frequency of fungal infections similar to other pediatric reports ${ }^{15-17}$ albeit with higher ratio of mold to yeast infections. Aspergillus infections need to be prevented and treated optimally, as 33\% and $43 \%$ of invasive aspergillosis during the second and third period had fatal outcomes.

The risk of infection varies with the underlying reason for the HSCT. ${ }^{18}$ However, we did not find the rate of viral or bacterial infections to differ according to the underlying illness. Although there was an association between the type of underlying illness and the rate of fungal infections, our objective was to describe the frequency rather than to identify risk factors for different infections. Therefore, we did not control for other variables (i.e. demographic factors, conditioning regimen), preventing us from reporting the independent risk of infections according to type of underlying illness.

The main limitation of this report is its retrospective nature, limiting our ability to precisely assess the different outcomes and relying on the accuracy of recordkeeping. In addition, our ability to accurately diagnose some infections was lower in the early part of the study period, probably leading to an underestimate of the true rate of some infections, especially those with viral etiology.

In conclusion, this data provides a better insight into the epidemiology of infections during the different post-HSCT phases in children from developing countries and it highlights the high burden that infections impose. Although overall mortality is high, trends in time are reassuring. However, key factors needed to be implemented in order to reduce morbidity and mortality in this population such as strict antibiotic stewardship strategies, better antiviral treatments (i.e. specific antiviral cytotoxic $\mathrm{T}$ lymphocyte therapy with donor T-cells), robust immune monitoring and better prevention or treatment of GVHD resulting in lower immunosuppression, among others.

\section{References}

1. Ardura MI. Overview of Infections Complicating Pediatric Hematopoietic Cell Transplantation. Infect Dis Clin North Am.2018;32(1):237-252.

2. Cesar-Arce A, Volkow-Fernandez P, Valero-Saldana LM, Acosta-Maldonado B, Vilar-Compte D, CornejoJuarez P. Infectious Complications and Multidrug-Resistant Bacteria in Patients With Hematopoietic Stem Cell Transplantation in the First 12 Months After Transplant. Transplant Proc. 2017;49(6):1444-1448.

3. Zajac-Spychala O, Wachowiak J, Pieczonka A, et al. Bacterial infections in pediatric hematopoietic stem cell transplantation recipients: incidence, epidemiology, and spectrum of pathogens: report of the Polish Pediatric Group for Hematopoietic Stem Cell Transplantation. Transpl Infect Dis. 2016;18(5):690-698. 
4. Tomblyn M, Chiller T, Einsele H, et al. Guidelines for preventing infectious complications among hematopoietic cell transplantation recipients: a global perspective. Biol Blood Marrow Transplant.2009;15(10):1143-1238.

5. Lehrnbecher T, Robinson P, Fisher B, et al. Guideline for the Management of Fever and Neutropenia in Children With Cancer and Hematopoietic Stem-Cell Transplantation Recipients: 2017 Update. J Clin Oncol. 2017;35(18):2082-2094.

6. Boeckh M, Ljungman P. How we treat cytomegalovirus in hematopoietic cell transplant recipients. Blood. 2009;113(23):5711-5719.

7. Feghoul L, Chevret S, Cuinet A, et al. Adenovirus infection and disease in paediatric haematopoietic stem cell transplant patients: clues for antiviral pre-emptive treatment. Clin Microbiol Infect.2015;21(7):701-709.

8. Srinivasan A, Klepper C, Sunkara A, et al. Impact of adenoviral stool load on adenoviremia in pediatric hematopoietic stem cell transplant recipients. Pediatr Infect Dis J. 2015;34(6):562-565.

9. Warris A, Lehrnbecher T, Roilides E, Castagnola E, Bruggemann RJM, Groll AH. ESCMID-ECMM guideline: diagnosis and management of invasive aspergillosis in neonates and children. Clin Microbiol Infect.2019;25(9):1096-1113.

10. Slade M, Goldsmith S, Romee R, et al. Epidemiology of infections following haploidentical peripheral blood hematopoietic cell transplantation. Transpl Infect Dis. 2017;19(1).

11. Magiorakos AP, Srinivasan A, Carey RB, et al. Multidrug-resistant, extensively drug-resistant and pandrug-resistant bacteria: an international expert proposal for interim standard definitions for acquired resistance. Clin Microbiol Infect. 2012;18(3):268-281.

12. De Pauw B, Walsh TJ, Donnelly JP, et al. Revised definitions of invasive fungal disease from the European Organization for Research and Treatment of Cancer/Invasive Fungal Infections Cooperative Group and the National Institute of Allergy and Infectious Diseases Mycoses Study Group (EORTC/MSG) Consensus Group. Clin Infect Dis.2008;46(12):1813-1821.

13. Ljungman P, Griffiths P, Paya C. Definitions of cytomegalovirus infection and disease in transplant recipients. Clin Infect Dis.2002;34(8):1094-1097.

14. Matthes-Martin S, Feuchtinger T, Shaw PJ, et al. European guidelines for diagnosis and treatment of adenovirus infection in leukemia and stem cell transplantation: summary of ECIL-4 (2011). Transpl Infect Dis. 2012;14(6):555-563.

15. Benjamin DK, Jr., Miller WC, Bayliff S, Martel L, Alexander KA, Martin PL. Infections diagnosed in the first year after pediatric stem cell transplantation. Pediatr Infect Dis J. 2002;21(3):227-234.

16. Gomez SM, Caniza M, Fynn A, et al. Fungal infections in hematopoietic stem cell transplantation in children at a pediatric children's hospital in Argentina. Transpl Infect Dis.2018;20(4):e12913.

17. Srinivasan A, Wang C, Srivastava DK, et al. Timeline, epidemiology, and risk factors for bacterial, fungal, and viral infections in children and adolescents after allogeneic hematopoietic stem cell transplantation. Biol Blood Marrow Transplant. 2013;19(1):94-101.

18. Michaels MG, Green M. Infections in pediatric transplant recipients: not just small adults. Infect Dis Clin North Am.2010;24(2):307-318.

\section{Hosted file}

Table_1 final.docx available at https://authorea.com/users/336973/articles/462648-infectiouscomplications-after-allogeneic-hematopoietic-stem-cell-transplantation-in-children-in-abone-marrow-transplant-unit-in-colombia

\section{Hosted file}


Table_2_final.docx available at https://authorea.com/users/336973/articles/462648-infectiouscomplications-after-allogeneic-hematopoietic-stem-cell-transplantation-in-children-in-abone-marrow-transplant-unit-in-colombia 